\title{
Development of real-time quantitative polymerase chain reaction assays to track treatment response in retinoid resistant acute promyelocytic leukemia
}

\section{Jelena V. Jovanovic ${ }^{1}$, Kristian Rennie ${ }^{2}$, Dominic Culligan ${ }^{3}$, Andrew Peniket $^{4}$, Anne Lennard $^{5}$, Justin Harrison ${ }^{6}$, Paresh Vyas ${ }^{7}$ and David Grimwade ${ }^{1 *}$}

${ }^{1}$ Cancer Genetics Laboratory, Department of Medical and Molecular Genetics, King's College London School of Medicine, London, UK

${ }^{2}$ GSTS Pathology, Guy's Hospital, London, UK

${ }^{3}$ Department of Haematology, Aberdeen Royal Infirmary, Aberdeen, UK

${ }^{4}$ Department of Haematology, John Radcliffe Hospital, Oxford, UK

${ }^{5}$ Department of Haematology, Royal Victoria Infirmary, Newcastle, UK

${ }^{6}$ Department of Haematology, Hemel Hempstead Hospital, Hemel Hempstead, UK

${ }^{7}$ Medical Research Council Molecular Haematology Unit, Weatherall Institute of Molecular Medicine, Oxford, UK

\section{Edited by:}

Marcos De Lima, MD Anderson Cancer Center, USA

Reviewed by:

Gheath Alatrash, University of Texas MD Anderson Cancer Center, USA

Ilana Renault Zalcberg, Instituto

Nacional de Cancer, Brazil

*Correspondence:

David Grimwade, Cancer Genetics Laboratory, Department of Medical and Molecular Genetics, Guy's Hospital, 8th Floor, Tower Wing, Great Maze Pond, London SE1 9RT, UK.

e-mail:david.grimwade@

genetics.kcl.ac.uk
Molecular detection of minimal residual disease (MRD) has become established to assess remission status and guide therapy in patients with ProMyelocytic Leukemia-RARA+ acute promyelocytic leukemia (APL). However, there are few data on tracking disease response in patients with rarer retinoid resistant subtypes of $A P L$, characterized by PLZF-RARA and STAT5b-RARA. Despite their rarity $(<1 \%$ of APL) we identified 6 cases (PLZF-RARA, $n=5$; STAT5b-RARA, $n=1$ ), established the respective breakpoint junction regions and designed reverse transcription-quantitative real-time polymerase chain reaction (RT-qPCR) assays to detect leukemic transcripts. The relative level of fusion gene expression in diagnostic samples was comparable to that observed in $t(15 ; 17)$ - associated $A P L$, affording assay sensitivities of $\sim 1$ in $10^{4}-10^{5}$. Serial samples were available from two PLZF-RARA APL patients. One showed persistent polymerase chain reaction positivity, predicting subsequent relapse, and remains in CR2, $\sim 11$ years post-autograft. The other, achieved molecular remission $(\mathrm{CRm})$ with combination chemotherapy, remaining in $\mathrm{CR} 1$ at 6 years. The STAT5b-RARA patient failed to achieve CRm following frontline combination chemotherapy and ultimately proceeded to allogeneic transplant on the basis of a steadily rising fusion transcript level. These data highlight the potential of RT-qPCR detection of MRD to facilitate development of more individualized approaches to the management of rarer molecularly defined subsets of acute leukemia.

\section{Keywords: minimal residual disease, acute myeloid leukemia}

\section{INTRODUCTION}

Acute promyelocytic leukemia (APL) is characterized by rearrangements of the gene encoding retinoic acid receptor alpha $(\mathrm{RAR} \alpha)$, which is most commonly fused to the ProMyelocytic Leukemia $(P M L)$ gene consequent upon the $\mathrm{t}(15 ; 17)(\mathrm{q} 22 ; 21)$ (reviewed Mistry et al., 2003). In approximately $10 \%$ of APL cases the $t(15 ; 17)$ is not detected, due to cytogenetic failures, simple variant translocations involving $15 \mathrm{q} 22$ or $17 \mathrm{q} 21$ and another partner chromosome, or more complex rearrangements (Grimwade et al., 2000). The majority of these cases lacking the classic $t(15 ; 17)$ nevertheless still harbor an underlying PML-RARA fusion gene, while in $\sim 1-2 \%$ of cases presenting with APL an alternative fusion partner is involved (Grimwade et al., 2000). These include PLZF (ZBTB16), NPM1, NuMA, FIP1L1, and BCOR, formed as a result of the $\mathrm{t}(11 ; 17)(\mathrm{q} 23 ; \mathrm{q} 21), \mathrm{t}(5 ; 17)(\mathrm{q} 35 ; \mathrm{q} 21), \mathrm{t}(11 ; 17)(\mathrm{q} 13 ; \mathrm{q} 21)$, $\mathrm{t}(4 ; 17)(\mathrm{q} 12 ; \mathrm{q} 21)$, and $\mathrm{t}(\mathrm{X} ; 17)(\mathrm{p} 11 ; \mathrm{q} 21)$, respectively; while the PRKAR1A, and STAT5b genes are fused to RARA following rearrangements involving 17q (Chen et al., 1993; Redner et al., 1996; Wells et al., 1997; Arnould et al., 1999; Catalano et al., 2007;
Kondo et al., 2008; Yamamoto et al., 2010). The nature of the fusion partner has an important bearing on disease biology, particularly the likely response to molecularly targeted therapies in the form of all-trans retinoic acid (ATRA) and arsenic trioxide (ATO). ATRA sensitivity has been documented in APL subtypes involving PML, NPM1, NuMA, and FIP1L1 (reviewed Grimwade et al., 2010); whereas, PLZF-RAR $\alpha$ and STAT5b-RAR $\alpha$ have both been associated with primary resistance to retinoids and a poorer prognosis (Licht et al., 1995; Arnould et al., 1999; Dong and Tweardy, 2002). In the case of PLZF-RAR $\alpha$ associated APL with the $t(11 ; 17)(q 23 ; q 21)$, the retinoid insensitivity is compounded by expression of the reciprocal RAR $\alpha-P L Z F$ fusion product from the derivative chromosome 17 [der(17)], which functions as a transcriptional activator targeting PLZF-binding sites leading to upregulation of cellular retinoic acid binding protein I (CRABP1), which sequesters retinoic acid, limiting its access to the nucleus (Guidez et al., 2007). To date, sensitivity to arsenic has only been demonstrated in PML-RAR $\alpha$ positive APL, reflecting the capacity of ATO to bind directly to the PML moiety of the fusion 
protein inducing its degradation via the proteosome (Zhang et al., 2010).

Molecular diagnostics to establish the nature of the fusion partner are therefore important for appropriate management, but in addition the application of sensitive minimal residual disease (MRD) assays to track treatment response has been found to be clinically useful in patients with PML-RAR $\alpha+$ disease, with previous studies showing that achievement of molecular remission $(\mathrm{CRm})$ as determined by qualitative or quantitative polymerase chain reaction (PCR) assays (with a sensitivity of 10-4) is a prerequisite for long-term remission and disease cure (reviewed Sanz et al., 2009; Grimwade and Tallman, 2011). These assays when applied at the post-consolidation timepoint are not sufficiently sensitive to identify all patients destined to relapse (Grimwade et al., 1996; Burnett et al., 1999). However, sequential molecular monitoring studies have shown that in patients who achieve $\mathrm{CRm}$, recurrence of PCR positivity heralds disease relapse (Diverio et al., 1998; Jurcic et al., 2001). Prediction is further refined by the use of reverse transcription-quantitative real-time PCR (RT-qPCR) which enables parallel quantification of endogenous control genes (e.g., Abelson, $A B L$ ) and leukemic transcripts, such that poor quality samples that could otherwise give rise to "false negative" results can be more reliably identified (Grimwade et al., 2009). Importantly, it also provides data on the kinetics of disease relapse, informing development of optimized MRD monitoring schedules (reviewed Freeman et al., 2008).

A significant complication of relapsed APL is death from hemorrhage due to the associated coagulopathy (Sanz et al., 2009). Therefore, Italian GIMEMA and Spanish PETHEMA groups explored the use of serial MRD monitoring as a tool to identify patients with impending relapse of APL (based upon persistent PCR positivity during therapy or recurrent PCR positivity in patients showing an initial response) to guide pre-emptive therapy to prevent disease progression (Lo Coco et al., 1999; Esteve et al., 2007). These studies, which were conducted before the availability of ATO for the treatment of relapse, suggested a survival benefit for early treatment intervention. More recently, we have shown in the Medical Research Council (MRC) AML15 trial that sequential monitoring using standardized RT-qPCR assays [developed within the Europe Against Cancer (EAC) program; Gabert et al., 2003)] provides the most powerful independent prognostic factor in APL (Grimwade et al., 2009). In addition we clearly demonstrated that these assays could be used to pinpoint particular patients destined to relapse, allowing successful delivery of pre-emptive therapy (Grimwade et al., 2009). This led to a significant reduction in the rate of frank relapse and improved survival, which was most marked in patients with high risk disease, i.e., with presenting white blood cell count above $10 \times 10^{9} / 1$. Moreover, we have shown that use of MRD monitoring to allow early deployment of ATO is associated with a significant reduction in treatment-related complications - substantially decreasing the risk of hyperleukocytosis and the associated lifethreatening differentiation syndrome (Grimwade et al., 2009). Accordingly molecular monitoring of MRD has become widely recognized as a standard component of care for patients with PML-RARA+ APL, as reflected in recent disease guidelines (Sanz et al., 2009).
While treatment is increasingly being tailored to the needs of individual patients, there are virtually no data on molecular monitoring in PLZF-RAR $\alpha$ and STAT5b-RAR $\alpha$ associated APL, which have been associated with a poorer prognosis. We have developed sensitive RT-qPCR assays suitable for tracking treatment response in these patients and which could be used to assess novel therapeutic approaches in retinoid insensitive disease.

\section{MATERIALS AND METHODS PATIENTS}

Our laboratory has served as the reference center for molecular diagnosis of APL for successive MRC/National Cancer Research Institute (NCRI) trials since 1994 and also receives samples for diagnosis and MRD monitoring from non-trial patients from across the UK (Burnett et al., 1999; Grimwade et al., 2009). To date, we have identified six cases of morphologically suspected APL presenting in the UK that lacked the $\mathrm{t}(15 ; 17)$ and were subsequently found to have an underlying PLZF-RARA $(n=5)$ or STAT5b-RARA $(n=1)$ fusion (Table 1). These include a previously unreported case (UPN 5) with the $\mathrm{t}(11 ; 17)(\mathrm{q} 23 ; \mathrm{q} 21)$ giving rise to the PLZF-RARA fusion, treated within the UK MRCAML12 trial. Clinical details of the APL patient with the STAT5b-RAR $\alpha$ fusion, who presented with pancytopenia and intracardiac thrombus have recently been described (Cahill et al., 2011). Samples were taken for molecular analysis following informed patient consent in accordance with the Declaration of Helsinki and the study was subject to Local Research Ethics Committee approval (St Thomas' Hospital Research Ethics Committee ref 06/Q0702/140).

\section{CHARACTERIZATION OF APL FUSION PARTNER}

Total RNA was extracted using the TRIzol reagent (Invitrogen Ltd., UK) according to the manufacturer's instructions, and $2 \mu \mathrm{g}$ were used for cDNA synthesis with random hexamers (Invitrogen Ltd., UK) and either M-MLV or SuperScript II reverse transcriptases (both Invitrogen Ltd., UK). In four cases with documented $\mathrm{t}(11 ; 17)$ (q23;q21) on diagnostic cytogenetic assessment, diagnostic samples were screened for expression of PLZF-RARA and reciprocal RARA-PLZF fusion transcripts by nested RT-PCR, as previously described (Grimwade et al., 1997). In 2 patients with simple variant translocations, i.e., $\mathrm{t}(7 ; 17)(\mathrm{q} 36 ; \mathrm{q} 21)$ and $\mathrm{t}(3 ; 17)(\mathrm{q} 26 ; \mathrm{q} 21)$ in UPN4 and UPN6, respectively, $5^{\prime}$ rapid amplification of cDNA ends (RACE) PCR was performed to identify the RARA fusion partner, using $2 \mu \mathrm{g}$ of total RNA and the $5^{\prime} / 3^{\prime}$ RACE Kit, second generation (Roche Diagnostics Ltd., UK) according to the manufacturer's instructions. First-strand cDNA was synthesized from $2 \mu \mathrm{g}$ of total RNA using an antisense gene-specific primer located in RARA exon 4 (SP1, 5' -CGGTGACACGTGTACACCATGTTC$3^{\prime}$ ) and the homopolymeric A-tail was added to its $3^{\prime}$ end as per manufacturer's instructions. Tailed cDNA was then amplified by PCR using a second gene-specific primer located in RARA exon 4 upstream of the SP1 primer (SP2, 5'TGGATGCTGCGGCGGAAGAAGC-3'), and the supplied Oligo dT-anchor primer (5'-GACCACGCGTATCGATGTCGAC $(\mathrm{T}){ }_{16} \mathrm{~V}$ $3^{\prime}$, where $\mathrm{V}=\mathrm{A}, \mathrm{C}$ or $\mathrm{G}$ ) which binds to the $5^{\prime}$ end of the poly(A)-tail. First round PCR product was then used as a template in a second PCR reaction with a nested PCR primer (SP3, 5'-CCATAGTGGTAGCCTGAGGACTTG-3') 
Table 1 | Clinical details and disease characteristics of patients with PLZF-RARA or STAT5b-RARA associated APL.

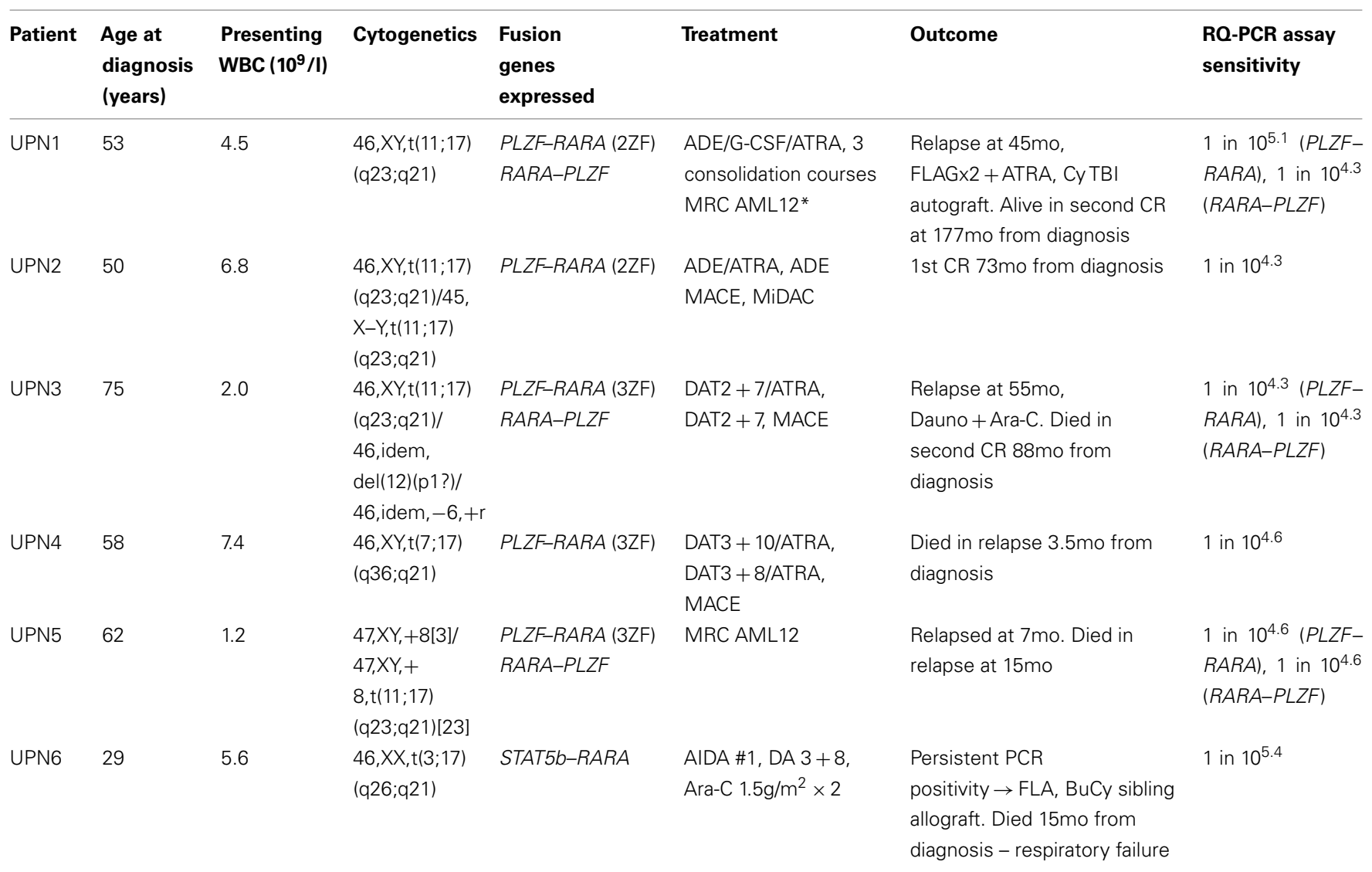

UPN5 is a previously unreported case. Clinical details and information regarding further molecular characterization of UPNs1-4 has been reported elsewhere (Culligan et al., 1998; Grimwade et al., 1997; Grimwade et al., 2000; Guidez et al., 2007). Details of the clinical presentation of the STAT5b-RARA case (UPN6) have been described previously (Cahill et al., 2011). *Details of the MRC AML12 protocol have been published previously (Burnett et al., 1999). Dauno, daunorubicin; Ara-C, cytosine arabinoside.

located in RARA exon 3 and the PCR anchor primer (5'GACCACGCGTATCGATGTCGAC-3') from the kit which anneals to the sequence introduced by the non-T portion of the Oligo $\mathrm{d}(\mathrm{T})$-anchor primer in the previous PCR round. Upon visualization in $1 \%$ agarose gels, purified $5^{\prime}$ RACE PCR products were cloned using the pGEM-T Easy Vector System (Promega, UK) and identified by sequence analysis (Figure 1). Breakpoint location was further verified by sequencing of nested RT-PCR products which were obtained from independent RNA aliquots.

\section{DEVELOPMENT OF RT-qPCR ASSAYS FOR APL FUSION TRANSCRIPTS}

The assay designs to amplify PLZF-RARA and STAT5b-RARA fusion transcripts were adapted from the standardized $P M L-$ RARA assay developed in the EAC program (Gabert et al., 2003), using the EAC probe and reverse primer located in $R A R A$ exon 3 in conjunction with newly designed forward primers located within PLZF (exon 3 or 4, depending upon the breakpoint) and STAT5b (exon 15), respectively (Figure 2; Table 2). In addition, reciprocal RARA-PLZF transcripts expressed from the $\operatorname{der}(17)$ were detected using a common forward primer and probe located in $R A R A$ exon 2, which were previously described for amplification of reciprocal $R A R A-P M L$ transcripts in APL with the classic $\mathrm{t}(15 ; 17)$
(Grimwade et al., 2009), used in conjunction with newly designed reverse primers located in PLZF exons 4 and 5, according to patient breakpoint (Figure 2; Table 2). Assays were designed using Primer Express software (Applied Biosystems, Warrington, UK). RT-qPCR reactions were run on the ABI7900 platform under the standard EAC conditions (Gabert et al., 2003), with expression of leukemic fusion transcripts normalized to the $A B L$ control gene using the $\Delta \mathrm{Ct}$ method, as described previously (Flora and Grimwade, 2004; Grimwade et al., 2009). All assays were confirmed to be fusion transcript specific based on lack of detectable amplification in normal control $(n=5)$ or diagnostic PML-RARA+APL $(n=5)$ blood and bone marrow (BM) samples. RT-qPCR assay sensitivity was calculated, based upon the level of expression of leukemic transcripts in the diagnostic sample in relation to the $A B L$ control gene, as described (Freeman et al., 2008; Grimwade et al., 2009). Assays were run in triplicate; amplification in at least two of three replicates with Cycle Threshold (Ct) values $\leq 40$ (threshold 0.05) was required to define a result as PCR positive for the fusion transcript in question, according to EAC criteria (Gabert et al., 2003). No-template controls (NTCs) for each assay were run in duplicate to exclude possible contamination, while patients' diagnostic samples served as positive controls. 

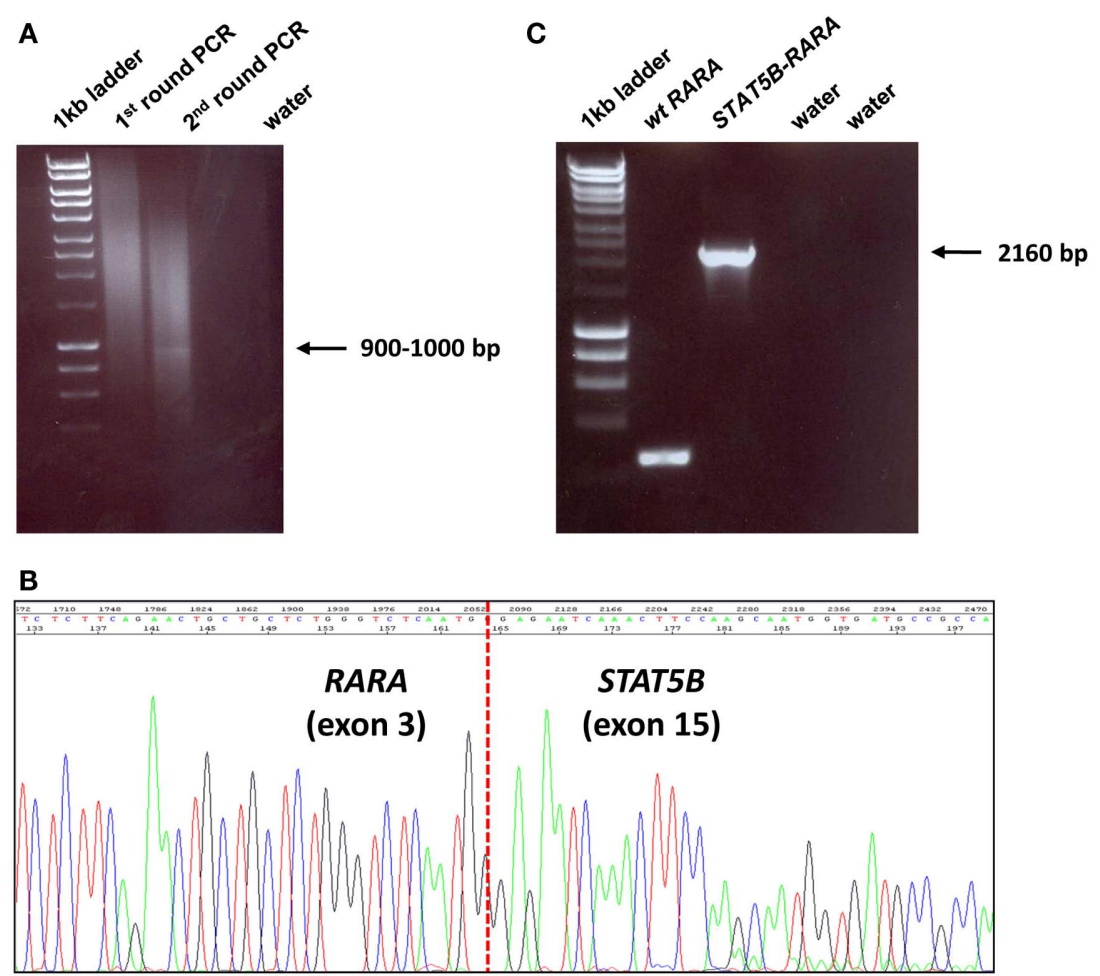

FIGURE 1 | Identification of STAT5b-RARA fusion underlying APL in UPN6 with $\mathbf{t}(3 ; 17)(\mathbf{q} 26 ; \mathbf{q} 21)$ variant translocation. (A) $5^{\prime}$ RACE was undertaken, which showed a weak band on second round PCR; the amplification product was cloned, sequenced and found to be a fusion between STAT5b exon 15 and RARA exon 3 (B), in accordance with the breakpoints identified in 4 previously reported cases with this rearrangement (Arnould et al., 1999; Kusakabe et al., 2008; Iwanaga et al., 2009; Qiao et al., 2011). Detection of STAT5b-RARA fusion transcripts was confirmed by nested RT-PCR using a fresh aliquot of RNA (C)
MRD level in follow-up samples was calculated using the $\Delta \Delta \mathrm{Ct}$ method as described by Beillard et al. (2003). Briefly, the difference in expression between the fusion transcript (FT) and $A B L$ in a follow-up (FUP) sample $\left(\Delta \mathrm{Ct}_{\mathrm{FUP}}=\mathrm{Ct}_{\mathrm{FT}}-\mathrm{Ct}_{\mathrm{ABL}}\right)$ was normalized to the difference between their expression at diagnosis $\left(\mathrm{D}_{\mathrm{x}}\right)\left(\Delta \mathrm{Ct}_{\mathrm{Dx}}=\mathrm{Ct}_{\mathrm{FT}}-\mathrm{Ct}_{\mathrm{ABL}}\right)$ using the following formula: $10^{[(\Delta \mathrm{CtFUP}-\Delta \mathrm{CtDx}) /-3.5]}$, where -3.5 represents the mean slope observed in the EAC program for plasmid standard curves (Beillard et al., 2003). Persistent PCR positivity was defined by the presence of leukemic transcripts throughout frontline therapy including the post-consolidation timepoint. CRm was defined as lack of detection of leukemic fusion transcripts in a BM sample affording a sensitivity of at least 1 in $10^{4}$.

\section{RESULTS}

\section{MOLECULAR CHARACTERIZATION OF APL CASES WITH ALTERNATIVE FUSION PARTNERS}

Molecular analysis was undertaken in six cases of PML-RARA negative APL. In four cases (UPN1-3, UPN5), cytogenetics showed the $\mathrm{t}(11 ; 17)$ (q23;q21), and presence of a PLZF/RARA rearrangement was confirmed by conventional nested RT-PCR (Table 1). In two cases (UPN4, UPN6) with $\mathrm{t}(7 ; 17)(\mathrm{q} 36 ; \mathrm{q} 21)$ and $\mathrm{t}(3 ; 17)(\mathrm{q} 26 ; \mathrm{q} 21)$ we postulated occurrence of a novel APL fusion; however, in both cases $5^{\prime}$ RACE revealed involvement of a known fusion partner, i.e., PLZF and STAT5b, respectively (Table 1; Figures 1 and
2), which was confirmed by nested RT-PCR performed on fresh aliquots of RNA from the diagnostic samples. In two cases with PLZF/RARA rearrangements, the chromosome 11 breakpoint fell within PLZF intron 3, leading to retention of 2 zinc fingers (2ZF) in the PLZF moiety of the PLZF-RAR $\alpha$ fusion protein. In the other three patients, the PLZF breakpoint fell within intron 4, leading to inclusion of 3 zinc fingers (3ZF) in the PLZF component of PLZF-RAR $\alpha$ (Table 1). Reciprocal RARA-PLZF fusion transcripts were co-expressed in three of five cases (Table 1). In UPN6 with the STAT5b-RARA fusion, the breakpoint location within the $S T A T 5 b$ locus was found to be identical to that reported previously (Arnould et al., 1999; Kusakabe et al., 2008; Iwanaga et al., 2009; Qiao et al., 2011; Figure 1). In accordance with the findings reported in the index case (Arnould et al., 1999), reciprocal RARA-STAT5 $b$ transcripts were not detected in UPN6.

\section{DEVELOPMENT OF RT-qPCR ASSAYS TO TRACK TREATMENT RESPONSE IN PATIENTS WITH PLZF-RARA AND STAT5B-RARA ASSOCIATED APL}

In order to detect $P L Z F-R A R A$ and STAT5b-RARA transcripts by RT-qPCR, forward primers were designed to be used in conjunction with the common probe and reverse primer developed within the EAC program to amplify $P M L-R A R A$ fusion transcripts (Figure 2; Gabert et al., 2003). To amplify reciprocal RARA-PLZF transcripts by RT-qPCR, reverse primers were designed within $P L Z F$ exon 4 or 5 (according to patient breakpoint location), used 


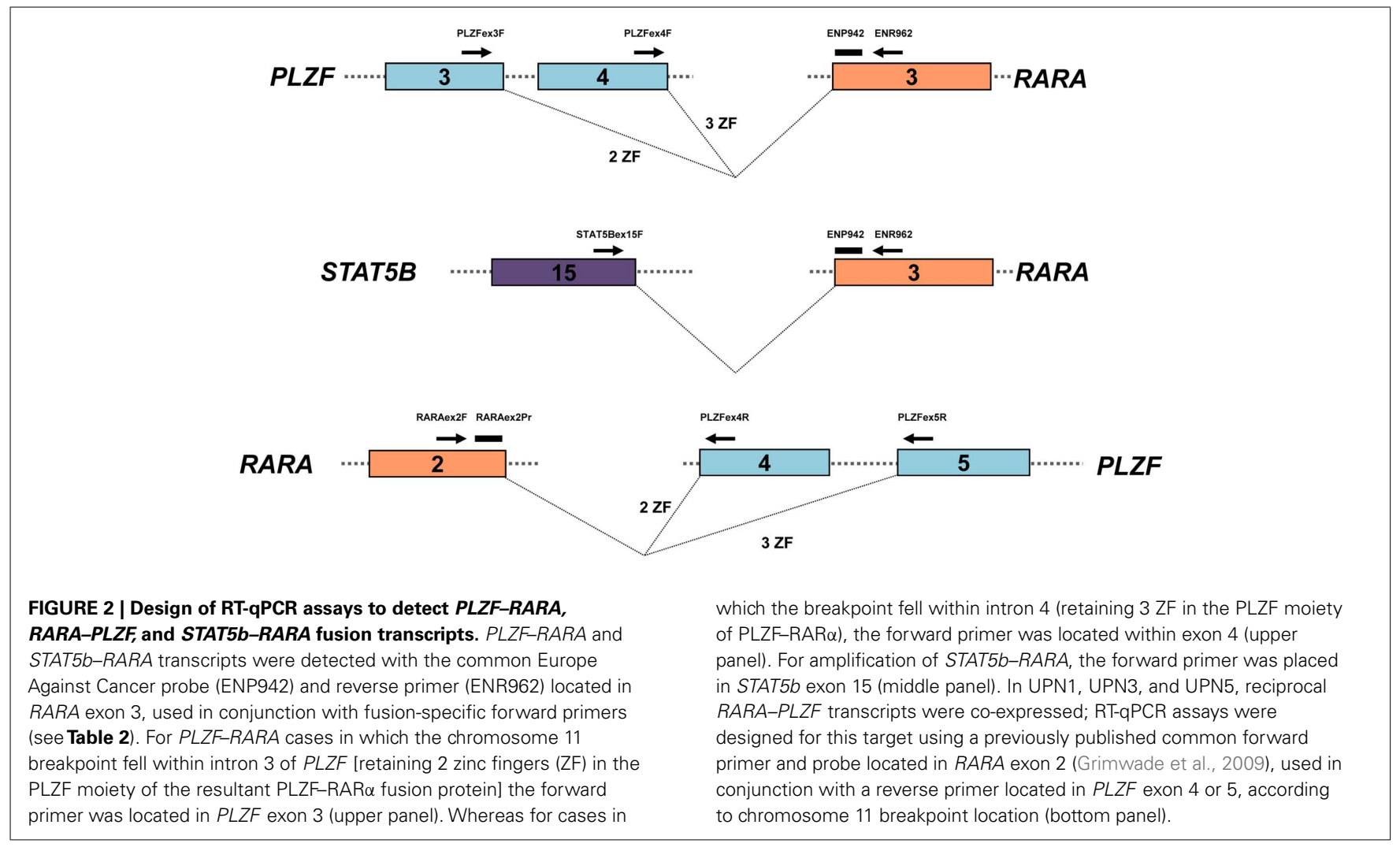

Table 2 | Primers and probes used to detect APL fusion transcripts.

\begin{tabular}{|c|c|}
\hline Primer/probe & Sequence $\left(5^{\prime}-3^{\prime}\right)$ \\
\hline \multicolumn{2}{|c|}{ PLZF-RARA AND STAT5B-RARA } \\
\hline PLZFex3F & TGGATAGTTTGCGGCTGAGA \\
\hline PLZFex4F & GAGACACACAGGCAGACCCATA \\
\hline STAT5Bex15F & GCATCACCATTGCTTGGAAG \\
\hline ENR962* & GCTTGTAGATGCGGGGTAGAG \\
\hline ENP942* & FAM-AGTGCCCAGCCCTCCCTCGC-TAMRA \\
\hline \multicolumn{2}{|l|}{ RARA-PLZF } \\
\hline RARAex $2 F^{\ddagger}$ & CCCCTATGCTGGGTGGACT \\
\hline PLZFex4R & CACCGCACTGATCACAGACAA \\
\hline PLZFex5R & AGACAGAAGACGGCCATGTCA \\
\hline RARAex2Pr ${ }^{\ddagger}$ & FAM-CCGCCAGGCGCTCTGACCAC-TAMRA \\
\hline
\end{tabular}

Sequences of the primers and probes used to detect PLZF-RARA, RARA-PLZF, and STAT5b-RARA fusion transcripts.

*Europe Against Cancer common reverse primer and probe (Gabert et al., 2003). ${ }^{\ddagger}$ Published common forward primer and probe, used previously to amplify RARA-PML (Grimwade et al., 2009).

in conjunction with the common forward primer and probe both located within $R A R A$ exon 2 (Figure 2 ), which we have recently validated for amplification of reciprocal $R A R A-P M L$ transcripts in patients with $\mathrm{t}(15 ; 17)$ APL within the UK MRC AML15 trial (Grimwade et al., 2009). The relative expression of PLZF-RARA at diagnosis was comparable to that observed for PML-RARA transcripts in $\mathrm{t}(15 ; 17)$ associated APL (Grimwade et al., 2009). The $\Delta \mathrm{Ct}_{\mathrm{Dx}}$ ranged from -2 to +1 , corresponding to assay sensitivities for detection of PLZF-RARA transcripts of between 1 in $10^{4.3}$ and 1 in $10^{5.1}$ (Table 1). In the three patients who were informative for the reciprocal $R A R A-P L Z F$ assay, this was not found to improve the sensitivity to detect MRD as compared to detection of PLZF$R A R A$ alone (Table 1). In UPN6, STAT5b-RARA transcripts were found to be very highly expressed at diagnosis $\left(\Delta \mathrm{Ct}_{\mathrm{Dx}}=-3\right)$, affording an assay sensitivity of 1 in $10^{5.4}$.

In two PLZF-RARA patients (UPN1, UPN2), follow-up samples were available for analysis. Samples from UPN1 had originally been tested by conventional nested RT-PCR, with BMs taken at 5 and 10 months from diagnosis found to test PCR negative. However, in accordance with our experience with $P M L-R A R A+\mathrm{APL}$, the RT-qPCR assay afforded greater sensitivity, with PLZF-RARA transcripts detected at both timepoints (Figure 3A). Reciprocal RARA-PLZF transcripts were not detectable in these follow-up samples in accordance with the poorer sensitivity afforded by this assay (Figure 3A; Table 1). Failure to achieve CRm following frontline therapy predicted subsequent disease relapse, which occurred at 45 months from original diagnosis (Figure 3A). In UPN2, MRD monitoring was undertaken by RT-qPCR in real time; in this case, $\mathrm{CRm}$ was achieved with combination chemotherapy, $P L Z F$ $R A R A$ transcripts remained undetected in subsequent surveillance MRD samples and this patient is in ongoing remission of APL at 73 months (Figure 3A). UPN6 with STAT5b-RARA was also monitored by RT-qPCR in real time (Figure 3B); this patient exhibited a 2-log reduction in fusion transcripts (i.e., $10^{-2} \mathrm{MRD}$ level) following AIDA induction (ATRA + idarubicin). However, treatment response was much poorer than typically seen in $P M L-R A R A+$ APL (Grimwade et al., 2009), with no significant further decline in 


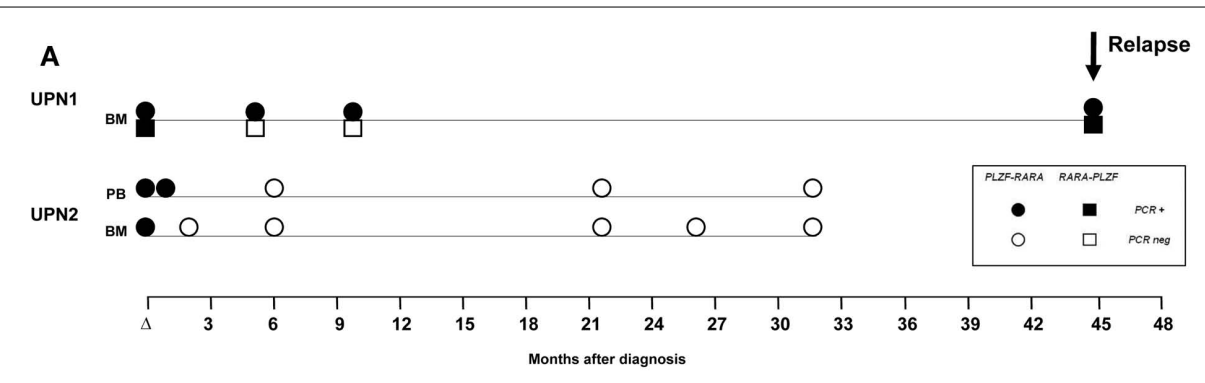

B

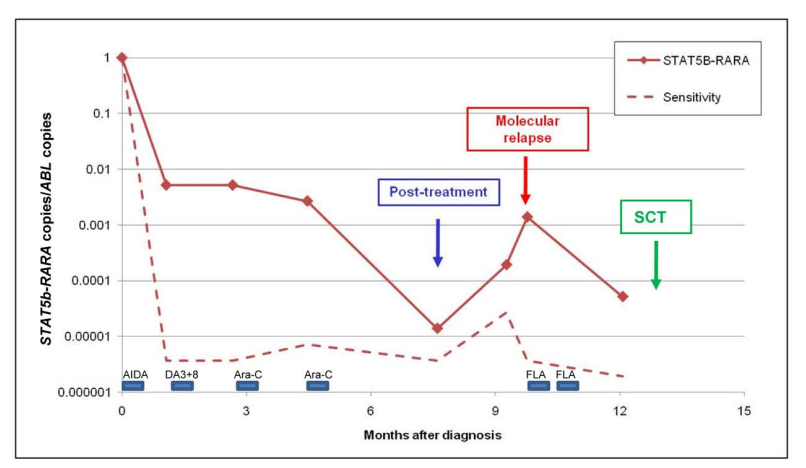

FIGURE 3 | Detection of minimal residual disease (MRD) by RT-qPCR assay in PLZF-RARA and STAT5b-RARA associated APL. (A) Serial samples were available from two patients (UPN1, UPN2) with $\mathrm{t}(11 ; 17)(\mathrm{q} 23 ; \mathrm{q} 21)$-associated APL. In UPN1, PLZF-RARA transcripts were still detectable at the post-treatment timepoint, predicting subsequent disease relapse. Reciprocal RARA-PLZF were not detectable in early follow-up samples, consistent with the poorer sensitivity of this assay in this patient (see Table 1). UPN2, who was not informative for RARA-PLZF, achieved molecular remission following frontline therapy and remains in ongoing remission of their leukemia. (B) Serial monitoring of STAT5b-RARA transcripts normalized to the $A B L$ control gene in UPN6. The patient failed to achieve molecular remission following frontline therapy and showed a rapidly rising fusion transcript level indicative of impending full blown relapse (labeled "molecular relapse"). The patient received two courses of Fludarabine and cytosine arabinoside (FLA) as pre-emptive therapy and then proceeded to a sibling myeloablative allogeneic stem cell transplant (SCT). fusion transcript level following two further courses of chemotherapy (DA3 + 8; cytarabine $\left.1.5 \mathrm{~g} / \mathrm{m}^{2}\right)$. A 2-log decline in fusion transcripts was documented following the fourth course of chemotherapy (cytarabine $1.5 \mathrm{~g} / \mathrm{m}^{2}$ ); however STAT5b-RARA transcripts remained detectable at the post-treatment timepoint and exhibited a steady rise of $\sim 2$-logs over the following 2 months. The patient was deemed to be in molecular relapse and received further therapy (Fludarabine, cytarabine) which led to a further decline in fusion transcripts. However, the patient never achieved a CRm and therefore proceeded to a myeloablative sibling donor allogeneic transplant with busulphan and cyclophosphamide conditioning, which was unfortunately complicated by respiratory failure, leading to the patient's demise while still in clinical remission.

\section{DISCUSSION}

Application of molecular monitoring by RT-qPCR to establish remission status and identify patients needing additional therapy to achieve disease cure is now firmly established as a key component of the management of patients with $P M L-R A R A+\mathrm{APL}$ (Sanz et al., 2009). However, there remains considerable uncertainty regarding the clinical utility of MRD monitoring in other forms of acute myeloid leukemia (AML). While there is evidence that RT-qPCR can be used to predict disease relapse in patients with nucleophosmin (NPM1) mutant AML (Schnittger et al., 2009; Krönke et al., 2011) and core binding factor (CBF) leukemia (Corbacioglu et al., 2010; Ommen et al., 2010), there are very limited data in patients with other molecularly defined subsets of disease.

To date, over 100 balanced chromosomal rearrangements which are considered to be primary events in leukemogenesis have been cloned (Mitelman et al., 2011). The characterization of the resulting chimeric fusion genes is not only important to achieve a greater understanding of disease biology, but has concomitantly yielded an extensive array of leukemia-specific targets that can effectively be used to track MRD by RT-qPCR. A number of genes (e.g., $M L L$, RUNX1, RARA, NUP98) are recurrently involved, fused to a range of potential partner genes. Depending upon breakpoint location, this allows common primers and probes located in the exon immediately adjacent to the breakpoint to be used in conjunction with an appropriate partner-gene specific primer to amplify the leukemic fusion transcript. In APL, translocation breakpoints consistently involve RARA intron 2, meaning that for cases with alternative fusion partners (e.g., PLZF, STAT5b, as described here), it is possible to use partner-specific forward primers in conjunction with an extensively validated probe and reverse primer located in $R A R A$ exon 3, that were designed in the EAC program (Gabert et al., 2003). Based on the expression of the fusion gene transcripts relative to the validated endogenous control gene $A B L$ in diagnostic samples, it was established that the sensitivity of the assays was comparable to those used in $P M L-R A R A+\mathrm{APL}$, capable of detecting MRD at a sensitivity of at least 1 in $10^{4}$. Due to the rarity of PLZF-RARA-associated APL, experience of MRD detection in 
patients with this subset of leukemia is extremely limited (Cassinat et al., 2006); nevertheless, the significance of the MRD results seems to parallel those observed in patients with classic $P M L-$ $R A R A+$ disease. In particular, $\mathrm{CRm}$ can be achieved with frontline therapy and is a prerequisite for disease cure.

As has been clearly demonstrated in PML-RARA+ APL, in order to reliably predict relapse it is important to adopt a sequential MRD monitoring approach (reviewed Grimwade and Tallman, 2011). This was applied in the patient with STAT5b-RARA+APL, showing a failure to achieve $\mathrm{CRm}$ following intensive frontline therapy. Based upon the rising transcript level, further therapy was given to prevent impending relapse followed by a sibling allogeneic transplant. This approach was based on published data showing that patients with $P M L-R A R A+$ APL with persistent PCR positivity can potentially be salvaged by allogeneic transplant (Lo-Coco et al., 2003; Grimwade et al., 2009; Kishore et al., 2010). However, unfortunately there was an unsuccessful outcome in our patient with STAT5b-RARA due to transplant-related complications.

Therefore in conclusion, we have used 5' RACE PCR to characterize simple variant translocations in APL, identifying cases involving the PLZF and STAT5b genes. Having defined breakpoint regions by sequence analysis, we adapted standardized RT-qPCR assays used for disease monitoring in patients with the classic $t(15 ; 17)$ in order to detect leukemic transcripts in these retinoid resistant subtypes of APL. As a consequence of the rarity of these disease entities, the number of cases analyzed was very small and

\section{REFERENCES}

Arnould, C., Philippe, C., Bourdon, V., Grégoire, M. J., Berger, R., and Jonveaux, P. (1999). The signal transducer and activator of transcription STAT5b gene is a new partner of retinoic acid receptor alpha in acute promyelocytic-like leukaemia. Hum. Mol. Genet. 8, 1741-1749.

Beillard, E., Pallisgaard, N., van der Velden, V. H., Bi, W., Dee, R., van der Schoot, E., Delabesse, E., Macintyre, E., Gottardi, E., Saglio, G., Watzinger, F., Lion, T., van Dongen, J. J., Hokland, P., and Gabert, J. (2003). Evaluation of candidate control genes for diagnosis and residual disease detection in leukemic patients using "real-time" quantitative reverse-transcriptase polymerase chain reaction (RQ-PCR) a Europe Against Cancer program. Leukemia 17, 2474-2486.

Burnett, A. K., Grimwade, D., Solomon, E., Wheatley, K., and Goldstone, A. H. (1999). Presenting white blood cell count and kinetics of molecular remission predict prognosis in acute promyelocytic leukemia treated with all-trans retinoic acid: result of the randomized MRC trial. Blood 93, 4131-4143.

Cahill, T. J., Chowdhury, O., Myerson, S. G., Ormerod, O., Herring, N., Grimwade, D., Littlewood, T., and Peniket, A. (2011). Myocardial

study of further patients is merited to identify thresholds that may be useful to predict risk of relapse. Nevertheless, this study highlights the potential of RT-qPCR to guide management in patients with infrequent recurring translocations for which there is currently a paucity of robust prognostic information on which to base treatment decisions, particularly with respect to the role of allogeneic transplant in first remission.

\section{ACKNOWLEDGMENTS}

We gratefully acknowledge Sarah Ryley, Anthony Moorman, and Kim Smith for cytogenetic data from UPN4, UPN5, and UPN6, respectively. We thank Susanna Akiki, Yashma Patel, Adele Timbs, and Anna Schuh for provision of diagnostic and follow-up samples for analysis, and Ciro Rinaldi and Robert Hills for clinical information. David Grimwade and Jelena V. Jovanovic gratefully acknowledge support from Leukaemia and Lymphoma Research (grants 0153, 04036), the European LeukemiaNet and the National Institute for Health Research for development and evaluation of MRD assays to predict outcome and guide treatment approach. This paper presents independent research funded by the National Institute for Health Research (NIHR) under its Programme Grants for Applied Research Programme (Grant Reference Number RPPG-0108-10093). The views expressed are those of the authors and not necessarily those of the NHS, the NIHR or the Department of Health. In addition David Grimwade is grateful for research funding from the Guy's and St. Thomas' Charity.

impact of minimal residual disease in CBFB-MYH11-positive acute myeloid leukemia. J. Clin. Oncol. 28, 3724-3729.

Culligan, D. J., Stevenson, D., Chee, Y. L., and Grimwade, D. (1998). Acute promyelocytic leukaemia with $\mathrm{t}(11 ; 17)(\mathrm{q} 23 ; \mathrm{q} 12-21)$ and a good initial response to prolonged ATRA and combination chemotherapy. $\mathrm{Br}$. J. Haematol. 100, 328-330.

Diverio, D., Rossi, V., Avvisati, G., De Santis, S., Pistilli, A., Pane, F., Saglio, G., Martinelli, G., Petti, M. C., Santoro, A., Pelicci, P. G., Mandelli, F., Biondi, A., and Lo-Coco, F. (1998). Early detection of relapse by prospective reverse transcriptasepolymerase chain reaction analysis of the PML/RARalpha fusion gene in patients with acute promyelocytic leukemia enrolled in the GIMEMAAIEOP multicenter "AIDA" trial. Blood 92, 784-789.

Dong, S., and Tweardy, D. J. (2002). Interactions of STAT5b-RARalpha, a novel acute promyelocytic leukemia fusion protein, with retinoic acid receptor and STAT3 signaling pathways. Blood 99, 2637-2646.

Esteve, J., Escoda, L., Martín, G., Rubio, V., Díaz-Mediavilla, J., González, M., Rivas, C., Alvarez, C., González San Miguel, J. D., Brunet, S., Tomás, J. F., Tormo, M., Sayas, M. J., Sánchez Godoy, P., Colomer, D., Bolufer, P.,
Sanz, M. A., and Spanish Cooperative Group PETHEMA. (2007). Outcome of patients with acute promyelocytic leukemia failing to front-line treatment with all-trans retinoic acid and anthracyclinebased chemotherapy (PETHEMA protocols LPA96 and LPA99): benefit of an early intervention. Leukemia 21, 446-452.

Flora, R., and Grimwade, D. (2004). Real-time quantitative RT-PCR to detect fusion gene transcripts associated with AML. Methods Mol. Med. 91, 151-173.

Freeman, S. D., Jovanovic, J. V., and Grimwade, D. (2008). Development of minimal residual disease directed therapy in acute myeloid leukemia. Semin. Oncol. 35, 388-400.

Gabert, J. A., Beillard, E., van der Velden, V. H. J., Bi, W., Grimwade, D., Pallisgaard, N., Barbany, G., Cazzaniga, G., Cayuela, J. M., Cavé, H., Pane, F., Aerts, J. L., De Micheli, D., Thirion, X., Pradel, V., González, M., Viehmann, S., Malec, M., Saglio, G., and van Dongen, J. J. (2003). Standardization and quality control studies of "real-time" quantitative reverse transcriptase polymerase chain reaction of fusion gene transcripts for residual disease detection in leukemia - a Europe Against Cancer program. Leukemia 17, 2318-2357. 
Grimwade, D., Biondi, A., Mozziconacci, M-J., Hagemeijer, A., Berger, R., Neat, M., Howe, K., Dastugue, N., Jansen, J., Radford-Weiss, I., Lo Coco, F., Lessard, M., Hernandez, J. M., Delabesse, E., Head, D., Liso, V., Sainty, D., Flandrin, G., Solomon, E., Birg, F., and Lafage-Pochitaloff, M. (2000). Characterization of acute promyelocytic leukemia cases lacking the classic $\mathrm{t}(15 ; 17)$ : results of the European working party. Blood 96, 1297-1308.

Grimwade, D., Gorman, P., Duprez, E., Howe, K., Langabeer, S., Oliver, F., Walker, H., Culligan, D., Waters, J., Pomfret, M., Goldstone, A., Burnett, A., Freemont, P., Sheer, D., and Solomon, E. (1997). Characterization of cryptic rearrangements and variant translocations in acute promyelocytic leukemia. Blood 90, 4876-4885.

Grimwade, D., Howe, K., Langabeer, S., Burnett, A., Goldstone, A., and Solomon, E. (1996). Minimal residual disease detection in acute promyelocytic leukemia by reverse-transcriptase PCR: evaluation of PML-RAR alpha and RAR alpha-PML assessment in patients who ultimately relapse. Leukemia 10 , 61-66.

Grimwade, D., Jovanovic, J. V., Hills, R. K., Nugent, E. A., Patel, Y., Flora, R., Diverio, D., Jones, K., Aslett, H., Batson, E., Rennie, K., Angell, R., Clark, R. E., Solomon, E., Lo-Coco, F., Wheatley, K., and Burnett, A. K. (2009). Prospective minimal residual disease monitoring to predict relapse of acute promyelocytic leukemia and to direct pre-emptive arsenic trioxide therapy. J. Clin. Oncol. 27, 3650-3658.

Grimwade, D., Mistry, A. R., Solomon, E., and Guidez, F. (2010). Acute promyelocytic leukemia: a paradigm for differentiation therapy. Cancer Treat. Res. 145, 219-235.

Grimwade, D., and Tallman, M. S. (2011). Should minimal residual disease monitoring be the standard of care for all patients with acute promyelocytic leukemia? Leuk. Res. 35, 3-7.

Guidez, F., Parks, S., Wong, H., Jovanovic, J. V., Mays, A., Gilkes, A. F., Mills, K. I., Guillemin, M. C., Hobbs, R. M., Pandolfi, P. P., de Thé, H., Solomon, E., and Grimwade, D. (2007). RARalpha-PLZF overcomes PLZF-mediated repression of CRABPI, contributing to retinoid resistance in $\mathrm{t}(11 ; 17)$ acute promyelocytic leukemia. Proc. Natl. Acad. Sci. U.S.A. 104, 18694-18699.
Iwanaga, E., Nakamura, M., Nanri, T., Kawakita, T., Horikawa, K., Mitsuya, H., and Asou, N. (2009). Acute promyelocytic leukemia harboring a STAT5B-RARA fusion gene and a G596V missense mutation in the STAT5B SH2 domain of the STAT5B-RARA. Eur. J. Haematol. 83, 499-501.

Jurcic, J. G., Nimer, S. D., Scheinberg, D. A., DeBlasio, T., Warrell, R. P. Jr., and Miller, W. H. Jr. (2001). Prognostic significance of minimal residual disease detection and PML/RAR-alpha isoform type: long-term follow-up in acute promyelocytic leukemia. Blood 98, 2651-2656.

Kishore, B., Stewart, A., Jovanovic, J., Craddock, C., and Grimwade, D. (2010). Arsenic trioxide and stem cell transplantation is an effective salvage therapy in patients with relapsed APL. Br. J. Haematol. 149(Suppl. 1), 22.

Kondo, T., Mori, A., Darmanin, S. Hashino, S., Tanaka, J., and Asaka, M. (2008). The seventh pathogenic fusion gene FIP1L1-RARA was isolated from a $\mathrm{t}(4 ; 17)$-positive acute promyelocytic leukemia. Haematologica 93, 1414-1416.

Krönke, J., Schlenk, R. F., Jensen, K. O., Tschürtz, F., Corbacioglu, A., Gaidzik, V. I., Paschka, P., Onken, S., Eiwen, K., Habdank, M., Späth, D., Lübbert, M., Wattad, M., Kindler, T., Salih, H. R., Held, G., Nachbaur, D., von Lilienfeld-Toal, M., Germing, U., Haase, D., Mergenthaler, H. G., Krauter, J., Ganser, A., Göhring, G., Schlegelberger, B., Döhner, H., and Döhner, K. (2011). Monitoring of minimal residual disease in NPM1-mutated acute myeloid leukemia: a study from the GermanAustrian Acute Myeloid Leukemia Study Group. J. Clin. Oncol. 29, 2709-2716.

Kusakabe, M., Suzukawa, K., Nanmoku, T., Obara, N., Okoshi, Y., Mukai, H. Y., Hasegawa, Y., Kojima, H., Kawakami, Y., Ninomiya, H., and Nagasawa, T. (2008). Detection of the STAT5B-RARA fusion transcript in acute promyelocytic leukemia with the normal chromosome 17 on G-banding. Eur. J. Haematol. 80, 444-447.

Licht, J. D., Chomienne, C., Goy, A., Chen, A., Scott, A. A., Head, D. R., Michaux, J. L., Wu, Y., DeBlasio, A., Miller, W. H. Jr, Zelenetz, A. D., Willman, C. L., Chen, Z., Chen, S.-J., Zelent, A., Macintyre, E., Veil, A., Cortes, J., Kantarjian, H., and Waxman, S. (1995). Clinical and molecular characterization of a rare syndrome of acute promyelocytic leukemia associated with translocation (11;17). Blood 85, 1083-1094.

Lo Coco, F., Diverio, D., Avvisati, G. Petti, M. C., Meloni, G., Pogliani, E. M., Biondi, A., Rossi, G., CarloStella, C., Selleri, C., Martino, B., Specchia, G., and Mandelli, F. (1999). Therapy of molecular relapse in acute promyelocytic leukemia. Blood 94, 2225-2229.

Lo-Coco, F., Romano, A., Mengarelli, A., Diverio, D., Iori, A. P., Moleti, M. L., De Santis, S., Cerretti, R., Mandelli, F., and Arcese, W. (2003). Allogeneic stem cell transplantation for advanced acute promyelocytic leukemia: results in patients treated in second molecular remission or with molecularly persistent disease. Leukemia 17, 1930-1933.

Mistry, A. R., Pedersen, E. W., Solomon, E., and Grimwade, D. (2003). The molecular pathogenesis of acute promyelocytic leukaemia: implications for the clinical management of the disease. Blood Rev. 17, 71-97.

Mitelman, F., Johansson, B., and Mertens, F. (eds). (2011). Mitelman Database of Chromosome Aberrations, and Gene Fusions in Cancer. Available at: http://cgap.nci.nih. gov/Chromosomes/Mitelman

Ommen, H. B., Schnittger, S., Jovanovic, J. V., Ommen, I. B., Hasle, H., Østergaard, M., Grimwade, D., and Hokland, P. (2010). Strikingly different molecular relapse kinetics in NPM1c, PML-RARA, RUNX1RUNX1T1, and CBFB-MYH11 acute myeloid leukemias. Blood 115, 198-205.

Qiao, C., Zhang, S. J., Chen, L. J., Miao, K. R., Zhang, J. F., Wu, Y. J., Qiu, H. R., and Li, J. Y. (2011). Identification of the STAT5B-RARalpha fusion transcript in an acute promyelocytic leukemia patient without FLT3, NPM1, c-Kit and C/EBPalpha mutation. Eur. J. Haematol. 86, 442-446.

Redner, R. L., Rush, E. A., Faas, S. Rudert, W. A., and Corey, S. J. (1996). The $t(5 ; 17)$ variant of acute promyelocytic leukemia expresses a nucleophosmin-retinoic acid receptor fusion. Blood 87, 882-886.

Sanz, M. A., Grimwade, D., Tallman, M. S., Lowenberg, B., Fenaux, P., Estey, E. H., Naoe, T., Lengfelder, E., Büchner, T., Döhner, H., Burnett, A. K., and Lo-Coco, F. (2009) Management of acute promyelocytic leukemia: recommendations from an expert panel on behalf of the European LeukemiaNet. Blood 113, 1875-1891.

Schnittger, S., Kern, W., Tschulik, C., Weiss, T., Dicker, F., Falini,
B., Haferlach, C., and Haferlach, T. (2009). Minimal residual disease levels assessed by NPM1 mutation-specific RQ-PCR provide important prognostic information in AML. Blood 114, 2220-2231.

Wells, R. A., Catzavelos, C., and KamelReid, S. (1997). Fusion of retinoic acid receptor alpha to NuMA, the nuclear mitotic apparatus protein, by a variant translocation in acute promyelocytic leukaemia. Nat. Genet. 17, 109-113.

Yamamoto, Y., Tsuzuki, S., Tsuzuki, M. Handa, K., Inaguma, Y., and Emi, N. (2010). BCOR as a novel fusion partner of retinoic acid receptor alpha in a $\mathrm{t}(\mathrm{X} ; 17)(\mathrm{p} 11 ; \mathrm{q} 12)$ variant of acute promyelocytic leukemia. Blood 116, 4274-4283.

Zhang, X. W., Yan, X. J., Zhou, Z. R., Yang, F. F., Wu, Z. Y., Sun, H. B., Liang, W. X., Song, A. X., LallemandBreitenbach, V., Jeanne, M., Zhang, Q. Y., Yang, H. Y., Huang, Q. H., Zhou, G. B., Tong, J. H., Zhang, Y., Wu, J. H., Hu, H. Y., de Thé, H., Chen, S. J., and Chen, Z. (2010). Arsenic trioxide controls the fate of the PML-RARalpha oncoprotein by directly binding PML. Science 328, 240-243.

Conflict of Interest Statement: The authors declare that the research was conducted in the absence of any commercial or financial relationships that could be construed as a potential conflict of interest.

Received: 31 July 2011; paper pending published: 20 August 2011; accepted: 28 September 2011; published online: 25 October 2011.

Citation: Jovanovic JV, Rennie $K$, Culligan D, Peniket A, Lennard A, Harrison J, Vyas $P$ and Grimwade $D$ (2011) Development of real-time quantitative polymerase chain reaction assays to track treatment response in retinoid resistant acute promyelocytic leukemia. Front. Oncol. 1:35. doi: 10.3389/fonc.2011.00035

This article was submitted to Frontiers in Hematology Oncology, a specialty of Frontiers in Oncology.

Copyright (C) 2011 Jovanovic, Rennie, Culligan, Peniket, Lennard, Harrison, Vyas and Grimwade. This is an openaccess article subject to a non-exclusive license between the authors and Frontiers Media SA, which permits use, distribution and reproduction in other forums, provided the original authors and source are credited and other Frontiers conditions are complied with. 